\title{
Math-Net.Ru
}

В. Г. Михайлов, Неравенства для числа высоковероятных исходов полиномиальной схемы, Дискрет. матем., 1998, том 10, выпуск 2, 45-51

DOI: https://doi.org/10.4213/dm422

Использование Общероссийского математического портала Math-Net.Ru подразумевает, что вы прочитали и согласны с пользовательским соглашением http://www.mathnet.ru/rus/agreement

Параметры загрузки:

IP : 3.89 .197 .203

26 апреля 2023 г., 10:25:37

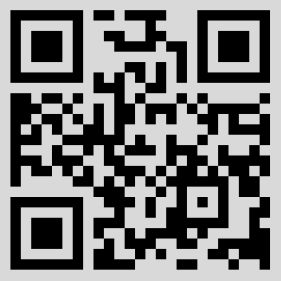




\title{
Аискретная математика
}

том 10 выпуск $2 * 1998$

\section{Неравенства для числа высоковероятных исходов полиномиальной схемы}

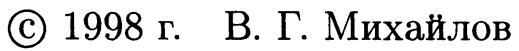

\begin{abstract}
В работе получено семейство оценок снизу для числа $w(x)$ исходов дискретной случайной величины, суммарная вероятность которых не меньше некоторого заданного числа $x$. На примерах показано, что при определенных условиях эти неравенства позволяют оценить функцию $w(x)$ с достаточно высокой точностью.

Работа выполнена при поддержке Российского фонда фундаментальных исследований, гранты 96-01-00531, 96-15-96092.
\end{abstract}

Пусть имеется дискретная вероятностная схема с $N$ элементарными исходами и вероятностями $p_{1}, \ldots, p_{N}$ исходов, $p_{1}+\ldots+p_{N}=1$. В теории случайных размещений такую схему обычно называют полиномиальной (см. [1]). Нам удобно считать, что $p_{1} \geqslant \ldots \geqslant p_{N}$. Положим

$$
w(x)=-\min \left\{k: p_{1}+\ldots+p_{N} \geqslant x\right\}
$$

Величина $w(x)$ имеет смысл минимального числа исходов схемы, суммарная вероятность которых не меньше заданного числа $x$. Требуется вычислить или оценить число таких исходов. Интерес к сформулированной выше математической задаче связан с тем, что у сильно неравновероятного распределения основная масса вероятности оказывается сосредоточенной на сравнительно небольшом числе исходов. Это свойство в определенных обстоятельствах может оказаться весьма существенным.

Вычисление функции $w(x)$ при большом числе исходов $N$ представляет собой достаточно сложную вычислительную задачу. Поэтому значительный интерес представляют оценки функции $w(x)$, и особенно оценки этой функции снизу.

В настоящей работе получена целая серия нижних оценок.

Положим

$$
P_{\beta}=\sum_{i=1}^{N} p_{i}^{\beta} .
$$

Теорема 1. Пусть $0<x \leqslant 1, \alpha>0$. Тогда

$$
w(x) \geqslant\left(\frac{x^{1+\alpha}}{P_{1+\alpha}}\right)^{1 / \alpha} .
$$


Следствие 1. Пусть $0<x \leqslant 1$. Тогда

$$
w(x) \geqslant \sup _{\alpha>0}\left(\frac{x^{1+\alpha}}{P_{1+\alpha}}\right)^{1 / \alpha} .
$$

Замечание 1. Наиболее интересным частным случаем неравенства (2) является неравенство

$$
w(x) \geqslant \frac{x^{2}}{P_{2}} .
$$

Дело здесь не только в простоте получающегося выражения, но и в том, что характеристика $P_{2}$ довольно часто встречается в различных задачах теории случайных размещений, и среди $P_{1+\alpha}$ оценивается статистически наиболее просто.

Замечание 2. Неравенства (2)-(4) очевидным образом сохраняют свою силу и для схем со счетным числом исходов. Ниже, демонстрируя возможности этих неравенств, мы оценим число высоковероятных исходов для геометрического распределения и распределения Пуассона.

Замечание 3. Неравенства (2)-(4) не исчерпывают всех средств исследования функции $w(x)$ при большом числе исходов. В тех условиях, когда вероятности исходов доствточно просто вычисляются, удобно использовать метод статистического моделирования. В некоторых случаях функция $w(x)$ может быть оценена в терминах энтропии распределения (см. например, [2]). Мы затронем этот вопрос ниже в примере 5.

Теорема 1 является следствием более общего результата.

Теорема 2. Пусть функция $f$ неотрицательна, монотонно возрастает и непрерывно дифференцируема на отрезке $[0,1]$, причем $f^{\prime}(0)=0, f^{\prime}(x)<f^{\prime}(y)$, если $x<y$. Положим

$$
P(f)=\sum_{k=1}^{N} f\left(p_{k}\right) .
$$

Tогда

$$
w(x) \geqslant \min \{k: P(f) / k \geqslant f(x / k)\} .
$$

Перейдем к доказательствам. Далее выражение $f^{-1}$ обозначает функцию, обратную функции $f$.

Лемма 1. Пусть выполнены условия

$$
p_{1}+\ldots+p_{N}=1, \quad f\left(p_{1}\right)+\ldots+f\left(p_{N}\right)=F,
$$

где $f(p)$ - некоторая неотрицательная, строго возрастающая и непрерывно дифференцируемая на $[0,1]$ функция, причем $f^{\prime}(0)=0, f^{\prime}(x)<f^{\prime}(y)$, если $x<y$. Тогда при любом $k=1, \ldots, N$

$$
p_{1}+\ldots+p_{k} \leqslant k f^{-1}(F / k) .
$$


Доказательство. Заметим, что при наших предположениях о свойствах функции $f^{\prime}(p)$ выполняется неравенство $f\left(p_{1}\right)+\ldots+f\left(p_{N}\right) \leqslant N f(1 / N)$, причем равенство достигается в равновероятном случае. поэтому условия (6) допускают равновероятный случай только при $F=N f(1 / N)$. В этом случае $k f^{-1}(F / k)=k f^{-1}(N f(1 / N) / k)$. $\mathrm{C}$ другой стороны, благодаря возрастанию функции $f^{-1}$ с ростом значения аргумента,

$$
p_{1}+\ldots+p_{k}=k / N=k f^{-1}(f(1 / N)) \leqslant k f^{-1}(N f(1 / N) / k) .
$$

Значит, в равновероятном случае неравенство (7) выполняется. Далее мы считаем, что $F<N f(1 / N)$.

Воспользуемся методом множителей Лагранжа. При этом нам удобно отказаться от предположения ограниченности единицей и неотрицательности величин $p_{1}, \ldots, p_{N}$. Поэтому мы доопределим нашу функцию $f$ для значений, больших единицы, произвольным образом с сохранением указанных в условии леммы свойств (что, очевидно, возможно), и доопределим функцию $f$ для отрицательных значений аргумента равенством $f(p)=f(|p|)$. При таком задании полученная функция будет непрерывно дифференцируема во всей области значений аргументов, выделяемой условиями (6). Рассмотрим задачу о поиске максимума функции $h\left(p_{1}, \ldots, p_{N}\right)=$ $p_{1}+\ldots+p_{k}$ при условиях

$$
g_{1}\left(p_{1}, \ldots, p_{N}\right)=0, \quad g_{2}\left(p_{1}, \ldots, p_{N}\right)=0
$$

где

$$
\begin{aligned}
& g_{1}\left(p_{1}, \ldots, p_{N}\right)=p_{1}+\ldots+p_{N}-1 \\
& g_{1}\left(p_{1}, \ldots, p_{N}\right)=f\left(p_{1}\right)+\ldots+f\left(p_{N}\right)-F
\end{aligned}
$$

В остальном величины $p_{1}, \ldots, p_{N}$ предполагаются произвольными, но, как мы уже отметили, среди них обязательно есть различные. Последнее влечет за собой линейную независимость строк матрицы

$$
D f=\left\|\frac{\partial}{\partial p_{j}} g_{i}\left(p_{1}, \ldots, p_{N}\right)\right\|=\left\|\begin{array}{cccc}
1 & 1 & \ldots & 1 \\
f\left(p_{1}\right) & f\left(p_{2}\right) & \ldots & f\left(p_{N}\right)
\end{array}\right\|
$$

во всей области, удовлетворяющей условиям (6), и гарантирует, что метод множителей Лагранжа укажет все относительные экстремумы. В нашем случае при непрерывно дифференцируемых функциях $h, g_{1}, g_{2}$ и компактном многообразии в качестве области значений аргументов это даст выражения как для максимального, так и для минимального значения функции $h$.

Рассмотрим вспомогательную функцию $H=h+\lambda_{1} g_{1}+\lambda_{2} g_{2}$, и составим систему уравнений $\frac{\partial H}{\partial p_{j}}=0, j=1, \ldots, N$. Получим систему уравнений

$$
\begin{aligned}
1+\lambda_{1}+\lambda_{2} f^{\prime}\left(p_{j}\right) & =0, & & j=1, \ldots, k, \\
\lambda_{1}+\lambda_{2} f^{\prime}\left(p_{j}\right) & =0, & & j=k+1, \ldots, N .
\end{aligned}
$$

Из этой системы следует, что точки экстремума обладают свойством

$$
p_{1}=\ldots=p_{k}, \quad p_{k+1}=\ldots=p_{N}
$$


Благодаря этому свойству условия (6) переписываются в виде

$$
k p_{1}+(N-k) p_{N}=1, \quad k f\left(p_{1}\right)+(N-k) f\left(p_{N}\right)=F .
$$

Последнее равенство влечет соотношение $k f\left({ }_{1}\right) \leqslant F$, которое дает нам оценку $p_{1} \leqslant$ $f^{-1}(F / k)$. Следовательно, в любом экстремальном случае

$$
p_{1}+\ldots+p_{k}=k p_{1} \leqslant k f^{-1}(F / k) .
$$

Лемма доказана.

Доказательство теоремы 2. Из леммы следует, что

$$
w(x)=\min \left\{k: p_{1}+\ldots+p_{k} \geqslant x\right\} \geqslant \min \left\{k: k f^{-1}(F / k) \geqslant x\right\} .
$$

Отсюда получаем (5). Теорема 2 доказана.

Доказательство теоремъ 1. Чтобы получить неравенство (2), в оценке (5) достаточно взять $f(y)=y^{1+\alpha}$. Тогда получим оценку

$$
w(x) \geqslant \min \left\{k: \frac{P_{1+\alpha}}{k} \geqslant\left(\frac{x}{k}\right)^{1+\alpha}\right\}=\min \left\{k: k^{\alpha} \geqslant \frac{x^{1+\alpha}}{P_{1+\alpha}}\right\} .
$$

Отсюда следует неравенство (2). Теорема 1 доказана.

Перейдем к примерам.

Пример 1. Пусть задано равновероятное распределение на множестве $\{1, \ldots, N\}$. В этом случае $w(x)=] x N[$. Напомним, что $] a[=-[-a]-$ наименьшее целое число, не меньшее числа $a$. В этом случае $P_{1+\alpha}=N^{-\alpha}$. Поэтому правая часть неравенства (2) имеет вид

$$
N \sup _{\alpha>0} x^{(1+\alpha) / \alpha}=N x
$$

Таким образом, в данном случае оценка (2) совпадает с истинным значением функции $w(x)$ при целом $x N$, и отличается от истинного значения функции $w(x)$ на величину $1-\{x N\}<1$, если число $x N$ дробное.

Однако в неравновероятном случае достичь столь высокой точности при всех $x$ невозможно.

Пример 2. Пусть на множестве $\{1, \ldots, M+N\}$ заданы вероятности

$$
p_{1}=\ldots=p_{M}=\frac{p}{M}, \quad p_{M+1}=\ldots=p_{M+N}=\frac{q}{N}, \quad p+q=1 .
$$

Тогда

$$
w(x)= \begin{cases}] x M / p[, & 0<x \leqslant p \\ M+N-[(1-x) N / q], & p<x \leqslant 1\end{cases}
$$

Кроме того,

$$
P_{1+\alpha}=\frac{p^{1+\alpha}}{M^{\alpha}}+\frac{q^{1+\alpha}}{N^{\alpha}}
$$


Пусть наше распределение неравновероятно : $p / M>q / N$. Тогда при $\alpha \rightarrow 0$

$$
\left(P_{1+\alpha}\right)^{1 / \alpha}=\frac{p}{M}(1+o(1)) .
$$

Значит,

$$
\sup \left(\frac{x^{1+\alpha}}{P_{1+\alpha}}\right) \geqslant \frac{M x}{p}
$$

Таким образом, в этом случае при $x \leqslant p$ правая часть выражения (5) отличается от $w(x)$ менее, чем на единицу. Однако при больших значениях $x$ значения всех функций

$$
\left(\frac{x^{1+\alpha}}{P_{1+\alpha}}\right)=\left(x^{1+\alpha}\left(\frac{p^{1+\alpha}}{M^{\alpha}}+\frac{q^{1+\alpha}}{N^{\alpha}}\right)^{-1}\right)^{1 / \alpha} .
$$

заметно отличаются от $w(x)$. Мы не приводим соответствующих выражений из-за их громоздкости.

Теперь рассмотрим два классических распределения на множестве неотрицательных целых чисел.

Пример 3. Рассмотрим геометрическое распределение с параметром $p$. В этом случае

$$
p_{k}=(1-p)^{k-1}, \quad k=1,2, \ldots,
$$

значит,

$$
p_{1}+\ldots+p_{r}=1-(1-p)^{r}
$$

и

$$
\left.w(x)=\min \left\{r: 1-(1-p)^{r} \geqslant x\right\}=\right] \frac{\ln (1-x)}{\ln (1-p)}[
$$

Кроме того,

$$
P_{1+\alpha}=\frac{p^{1+\alpha}}{1-(1-p)^{1+\alpha}}
$$

Поэтому оценка (2) имеет вид

$$
w(x) \geqslant\left(\left(\frac{x}{p}\right)^{1+\alpha}\left(1-(1-p)^{1+\alpha}\right)\right)^{1 / \alpha}, \quad \alpha>0 .
$$

Выясним точность этих оценок. Нетрудно убедиться в том, что при $x \leqslant 1 / 2$

$$
\frac{\ln (1-x)}{\ln (1-p)} \leqslant \frac{x+x^{2}}{p}
$$


и, следовательно,

$$
w(x) \leqslant \frac{x+x^{2}}{p}+1
$$

в свою очередь, оценки (2) дают неравенства

$$
\begin{aligned}
w(x) & \geqslant \frac{x^{2}(2-p)}{p}, & \alpha & \alpha, \\
w(x) & \geqslant \frac{x^{3 / 2}}{p} \sqrt{3-3 p+p^{2}}, & \alpha & =2, \\
w(x) & \geqslant \frac{x}{p}, & \alpha & \rightarrow \infty .
\end{aligned}
$$

Последняя из этих оценок отличается при $p / 2 \leqslant x \leqslant 1 / 2$ от верхней оценки (10) не более чем в два раза. При $x>1 / 2$ точность оценивания снижается.

Пример 4. Рассмотрим пуассоновское распределение. Приведем результаты вычисления и оценивания функции $w(x)$ для пуассоновского распределения с параметром 10000000 . Выбор столь больного значения параметра объясняется следующим. Во-первых, чем больше значение параметра, тем сильнее проявляется концентрация распределения на высоковероятных исходах. Во-вторых, при выбранном значении параметра пуассоновское распределение практически не отличается от сгруппированного в целые точки нормального распределения с соответствующими значениями среднего и дисперсии.

В приводимой ниже таблице в первом столбце указаны значения параметра $x$, во втором - функции $w(x)$, а в пяти следующих - оценки, получаемые с помощью

\begin{tabular}{|c|c|c|c|c|c|c|c|}
\hline & \multicolumn{5}{|c|}{$\alpha$} & \multirow[b]{2}{*}{$\%$} \\
\hline$x$ & $w(x)$ & 1 & 2 & 3 & 4 & $\infty$ & \\
\hline$\overline{00,1}$ & $\overline{795}$ & $\overline{112}$ & $\overline{330}$ & $\overline{464}$ & $\overline{545}$ & $\overline{793}$ & 99,7 \\
\hline 0,2 & 1603 & 449 & 933 & 1168 & 1296 & 1585 & 98,9 \\
\hline 0,3 & 2437 & 1009 & 1714 & 2006 & 2152 & 2378 & 97,6 \\
\hline 0,4 & 3317 & 1794 & 2639 & 2943 & 3083 & 3171 & 95,6 \\
\hline 0,5 & 4266 & 2803 & 3688 & 3963 & 4075 & 3963 & 95,5 \\
\hline 0,6 & 5323 & 4037 & 4848 & 5054 & 5119 & 4756 & 96,2 \\
\hline 0,7 & 6555 & 5494 & 6110 & 6207 & 6206 & 5549 & 94,7 \\
\hline 0,8 & 8106 & 7176 & 7465 & 7417 & 7334 & 6341 & 92,1 \\
\hline 0,9 & 10403 & 9083 & 8907 & 8678 & 8497 & 7134 & 87,3 \\
\hline
\end{tabular}
неравенства (2) при $\alpha=1,2,3,4$ и $\alpha \rightarrow \infty$ соответственно. Полужирным выделены наилучшие значения оценок. В правом столбце указано, какую долю в процентах от истинного значения функции $w(x)$ составляет наилучшая из приведенных оценок.

Ясно, что отмеченная точность оценок имеет место не только для указанных в таблице значений $x$, но и в промежутках между ними. Таким образом, в рассмотренном примере серия оценок (2) (или, что то же самое, неравенство (3)) позволяет оценить функцию $w(x)$ при $x \leqslant 0,9$ не менее, чем с $87,3 \%$-й точностью.

Предыдущие примеры были призваны продемонстрировать точность предложенных оценок. Теперь коснемся вопроса их применимости. Приведенные примеры были специально подобраны так, чтобы непосредственное вычисление функции $w(x)$ 
не составляло труда. При этом определенную сложность представляло собой вычисление величин $P_{1+\alpha}$. Приведем теперь пример, в котором вычисление величин $P_{1+\alpha}$ много проще вычисления функции $w(x)$.

Пример 5. Рассмотрим неравновероятную полиномиальную схему с $m$ элементарными исходами и вероятностями исходов $q_{1}, \ldots, q_{m}, q_{1}+\ldots+q_{m}=1$. Пусть производится случайный зксперимент, состоящий из $s$ независимых испытаний в этой схеме и $Y=\left(X_{1}, \ldots, X_{s}\right)$ - исход этого зксперимента. Очевидно, что случайная величина $Y$ имеет неравновероятное полиномиальное распределение с $N=m^{s}$ элементарными исходами. Вероятности этих исходов легко вычисляются по вероятностям $q_{1}, \ldots, q_{m}$. Однако, если число $N=m^{s}$ очень велико (например, если велико число $s$ ), то вычисление функции $w(x)$ с помощью обычного упорядочивания вероятностей оказывается очень трудоемкой процедурой. Вместе с тем, при небольших значениях параметра $m$ вычисление величин $P_{1+\alpha}$ не составляет труда. В данном случае

$$
P_{1+\alpha}=Q_{1+\alpha}^{s}=\left(\sum_{k=1}^{m} q_{k}^{1+\alpha}\right)^{s} .
$$

Заметим, что (см., например, теорему 3 на с.233 в [2])

$$
\frac{\ln w(x)}{s} \rightarrow H=-\sum_{k=1}^{m} q_{k} \ln q_{k}, \quad 0<x<1 .
$$

Более того, нетрудно показать (см. [2], с.234), что

$$
\log w(x) \geqslant s H-u(s)+\ln x
$$

где $0<u(s)=o(s)$ при $s \rightarrow \infty$. Из-за неопределенного члена $u(s)$ оценка (12) непригодна для явного оценивания функции $w(x)$ при конкретных значениях параметров, но являются асимптотически точной.

Интересно сравнить оценку (12) с неравенством (2). Перейдем в последнем к логарифмам и подставим в него выражение (11):

$$
\ln w(x) \geqslant-\frac{s}{\alpha} \ln Q_{1+\alpha}+\frac{1+\alpha}{\alpha} \ln x .
$$

Из неравенства Йенсена (см., например, [3], с.15) следует соотношение $-\ln Q_{1+\alpha} \leqslant$ $\alpha H$. Оно обращается в равенство лишь при $q_{1}=\ldots=q_{m}=1 / m$, и лишь в этом случае оценка (13) является асимптотически точной.

Автор признателен А. М. Зубкову за ряд полезных замечаний.

\section{Список литературы}

1. Колчин В. Ф., Севастьянов Б. А., Чистяков В. П. Случайные размещеяия. Наука, Москва, 1976.

2. Боровков А. А. Курс теории вероятностей. Наука, Москва, 1972.

3. Петров В. В. Предельные теоремы для сумм независимых случайных величин. Наука, Москва, 1987. 